\title{
Colaboración de la Asociación Nacional de Cardiólogos de México y de la Sociedad Mexicana de Cardiología con la Universidad de Guanajuato. COVID-19 y personal de imagen cardiaca: Revisión de prácticas sanitarias al inicio de 2021
}

\author{
Collaboration between the National Association of Cardiologists of Mexico, the Mexican \\ Society of Cardiology, and the University of Guanajuato. COVID-19 and Cardiac Imaging \\ Personnel: Review of Sanitary Practices in Early 2021
}

Andrés Preciado-Anaya ${ }^{1 *}$, Isabel Carvajal-Juárez², Sara Llanos-Osuna ${ }^{3}$, Gabriela Meléndez-Ramírez ${ }^{4}$, Rafael Paz-Gómez ${ }^{5}$, Erik T. Kimura-Hayama ${ }^{6}$, Nelsy C. González-Ramírez ${ }^{6}$, Sergio G. Olmos-Temois ${ }^{7}$, Erasmo de la Peña-Almaguer ${ }^{8}$, Alejandro E. Macías-Hernández ${ }^{9}$, Óscar U. Preciado-Gutiérrez ${ }^{9}$, Sandra X. Bolaños-Hurtado ${ }^{10}$, Áyax N. Sobrino-Saavedra11,12; Alejandra de la Torre-Gascón ${ }^{12}$, Moisés Jiménez-Santos ${ }^{13}$, Adriana Puente-Barragán ${ }^{14}$, Luiz Ruiz-Monterrubio ${ }^{15}$ y José E. Jaramillo-Almaguer ${ }^{16}$

${ }^{1}$ Departamento de Cardiología Nuclear y Tomografía, Hospital Siena del Moral, Centro de investigación Cardimax y Hospital General de Zona \#

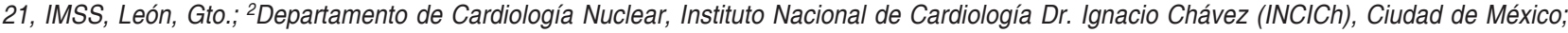
${ }^{3}$ Unidad de PET/CT, Clínica médica GOCA, Querétaro, Qro.; ${ }^{4}$ Departamento de Resonancia Cardiaca, INCICh, Ciudad de México; ${ }^{5}$ Servicio de Imagenología, Hospital Médica Campestre, León, Gto.; ${ }^{6}$ Servicio de Imagenología, CT Scanner Lomas altas, Ciudad de México; ${ }^{7}$ Servicio de Cardiología, Hospital General de Celaya, Celaya, Gto.; ${ }^{8}$ Instituto de Cardiología y Medicina Vascular del Instituto Tecnológico y de Estudios Superiores de Monterrey, Monterrey, N.L.; ${ }^{9}$ Departamento de Medicina y Nutrición, Universidad de Guanajuato, León, Gto.; ${ }^{10}$ Departamento de Radiología, Unidad Médica de Alta Especialidad, Hospital de especialidades N. ${ }^{0}$ 1, Centro Médico Nacional Bajío, León, Gto.; ${ }^{11}$ Instituto Cardiovascular de Mínima Invasión, Guadalajara, Jal.; ${ }^{12}$ Unidad de Patología Clínica, Hospital Real San José Valle Real, Guadalajara, Jal.; ${ }^{13}$ Servicio de Radiología, Unidad Médica de Alta Especialidad, Hospital de Cardiología del Centro Médico Nacional Siglo XXI, IMSS, Departamento de Tomografía, INCICh, Ciudad de México; ${ }^{14}$ Servicio de Cardiología Nuclear, Centro Médico Nacional 20 de noviembre, ISSSTE, Ciudad de México; ${ }^{15}$ Unidad PET/CT, Hospital Aranda de la Parra, León, Gto.; ${ }^{16}$ Servicio de Imagenología, Hospital Aranda de la Parra, León, Gto. México

\begin{abstract}
Resumen
Los capítulos de imagen de la Asociación Nacional de Cardiólogos de México (ANCAM) y de la Sociedad Mexicana de Cardiología (SMC), así como personal del Departamento de Medicina y Nutrición de la Universidad de Guanajuato, en conjunto con destacados expertos de la imagen cardiovascular en México, han colaborado en la revisión, análisis y ampliación de las diversas estrategias sanitarias publicadas en los primeros 15 meses de la pandemia de enfermedad por coronavirus 2019 (COVID-19) para realizar con seguridad los estudios de imagen cardiaca; esta actualización tiene como objetivo principal disminuir el riesgo de transmisión de la COVID-19 entre los pacientes y el personal de salud en los servicios de tomografía, resonancia y cardiología nuclear. Este trabajo se amplió con información suplementaria disponible sin costo en el sitio www.ancam-imagen.com.
\end{abstract}

Palabras clave: COVID-19. Prevención. Imagen cardiaca.

Correspondencia:

*Andrés Preciado Anaya

E-mail: cicamet@gmail.com
Disponible en internet: 19-03-2021 Arch Cardiol Mex. 2021;91(Supl):110-122 www.archivoscardiologia.com CC BY-NC-ND (http://creativecommons.org/licenses/by-nc-nd/4.0/). 


\begin{abstract}
The authors of the image chapters of the National Association of Cardiologists of Mexico (ANCAM) and the Mexican Society of Cardiology (SMC), as well as personnel from the Department of Medicine and Nutrition of the University of Guanajuato, together with prominent experts in cardiovascular imaging from Mexico, have collaborated in the review, analysis and expansion of the various health strategies published in the first year of the coronavirus disease 2019 (COVID-19) pandemic, to safely perform cardiac imaging studies. This update aims to reduce the risk of COVID-19 transmission among patients and health-care personnel in the CT, MRI, and nuclear cardiology services. This work was expanded with supplementary information available free of charge on the website www.ancam-imagen.com.
\end{abstract}

Key words: COVID-19. Prevention. Cardiac imaging.

\section{Introducción}

Los coronavirus son una extensa familia de virus que causan enfermedades infecciosas en animales y humanos, que van desde enfermedades leves hasta graves.

El 11 de marzo de 2020, la Organización Mundial de la Salud (OMS) declaró pandemia a la enfermedad por coronavirus 2019 (COVID 19) causada por el coronavirus 2 del síndrome respiratorio agudo grave (SARS-CoV-2) ${ }^{1}$.

Al final del año 2020, México registró una prolongada primera ola del brote pandémico, que no disminuyó de forma sostenida desde su inicio en nuestro país.

A escala mundial, la enfermedad se considera una de las mayores emergencias sanitarias de los últimos cien años por los principales organismos y sociedades médicas, debido a sus características definitorias de rapidez, gravedad, perturbación económica y social.

Por ahora y hasta que un porcentaje suficiente de la población esté vacunada o hasta disponer de fármacos más efectivos, la mejor forma de enfrentar la pandemia es reforzar la observancia y seguimiento estricto de las medidas de prevención para reducir o enlentecer la tasa de contagio. En septiembre de 2020, México tenía el mayor número de muertes entre profesionales de la salud a nivel mundial, seguido por los EE.UU. ${ }^{2}$, por lo que parece justificado revisar las medidas de seguridad descritas en la literatura con el objetivo de reforzar su difusión y aplicación de forma estricta en este grupo de alto riesgo, del que forma parte el personal que labora en los servicios de imagen cardiaca.

La creciente evidencia documentada sobre la evolución y transmisión ${ }^{3-8}$ de COVID-19 es incorporada casi de forma inmediata a guías y protocolos en actualización constante, que se aplican por instituciones y personal sanitario ${ }^{9-13}$ que manejan la enfermedad, incluida su prevención en portadores de patología cardiovascular ${ }^{14-16}$.
Un ejemplo de la rapidez con que evolucionan las recomendaciones para prevenir la COVID-19 es el cambio reciente en la percepción sobre la utilidad que el uso masivo y obligatorio del cubrebocas representa para limitar la transmisión aérea del virus, también dentro de las salas y gabinetes de imagen, que son espacios cerrados, donde los aerosoles emitidos por pacientes pueden durar suspendidos y viajar por más tiempo y distancia que lo supuesto al inicio del brote pandémico ${ }^{8,16}$. Un ejemplo más sobre la velocidad con que se adquiere nuevo conocimiento es el aparente alto porcentaje de complicaciones cardiacas observadas en pacientes recuperados de COVID-19 ${ }^{17}$.

Los capítulos de imagen 2018-2020, de la Asociación Nacional de Cardiólogos de México (ANCAM) y de la Sociedad Mexicana de Cardiología 2020-2022, así como el Departamento de Medicina y Nutrición de la Universidad de Guanajuato, en colaboración con algunos expertos del país, han analizado la evidencia publicada hasta la fecha por algunas de las sociedades y organismos especializados en imagen cardiaca más importantes del mundo ${ }^{18-22}$ sobre las estrategias de seguridad sanitaria usadas durante la realización de los estudios de imagen cardiaca. Se revisaron las recomendaciones para el personal y pacientes de servicios de imagen y algunas de ellas se adaptaron para contribuir a la disminución de la transmisión de COVID-19 y aumentar la seguridad en cada centro de trabajo. Las recomendaciones técnicas para escenarios clínicos específicos en tomografía computarizada por emisión de fotón único (SPECT), tomografía por emisión de positrones (PET), angiotomografía (CT) y resonancia magnética (RM) pueden consultarse en las publicaciones de las sociedades médicas especializadas correspondientes ${ }^{18-20}$ y en las tablas y figuras adaptadas de esas recomendaciones, traducidas al idioma español y clasificadas por grados de urgencia, disponibles en el material suplementario con información relacionada de interés, que se puede descargar 
Tabla 1. Recomendaciones básicas para personal en centros de imagen

a) Higiene frecuente de manos y no tocarse la cara

c) Uso de lentes y caretas plásticas

e) Cubrir con el pliegue del codo nariz y boca al estornudar o toser aún con mascarilla b) Uso de mascarillas apropiadas a cada situación clínica, vigile y exija usar mascarillas SIN VÁLVULA

d) Favorecer el intercambio aéreo y la ventilación en salas de diagnóstico antes de cada estudio, después de su adecuada limpieza

f) Evitar estancia prolongada en espacios concurridos o cerrados. Entre más tiempo permanezca en un espacio cerrado, mayor es el riesgo de contagio

g) Distancia "segura» de dos metros entre personas. No olvidar que: al hablar fuerte, gritar, toser, reír o jadear durante ejercicio físico, se generan aerosoles y estos dentro de espacios cerrados como las salas de imagen, viajan distancias mayores de 2 metros y permanecen por horas suspendidos en el aire ambiente ${ }^{8}$. Por lo tanto, en espacios cerrados la "distancia segura de 2 metros" debe considerarse asociada a este razonamiento. La principal forma de transmisión de SARS-CoV-2 parecen ser los aerosoles en espacios cerrados o mal ventilados. El personal de imagen debe portar siempre el equipo adecuado de protección

\section{Personal en centros de imagen con síntomas de infección respiratoria aguda}

Toma de muestra para SARS-CoV-2

Aislamiento domiciliario estricto

\section{POSITIVO}

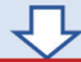

Regreso al centro de imagen 10 días después del inicio de síntomas + al menos 3 días sin fiebre, sin antipiréticos, ni síntomas respiratorios. PERO SI EL PACIENTE TUVO SÍNTOMAS POR MÁS DE 10 DÍAS, DEBERÁ REGRESAR 3 DÍAS DESPUÉS DE NO TENER SÍNTOMAS. Ejemplo: si un paciente duró 30 días con síntomas, debe regresar $30+3=33$ días después del inicio de síntomas. Pacientes asintomáticos: regresar 10 días después del resultado positivo para SARS CoV-2.

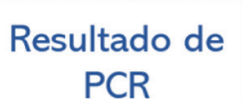

No es necesario un segundo examen de PCR antes de volver a trabajar.

Figura 1. Flujograma del manejo de personal con infección respiratoria aguda en gabinetes de imagen (adaptada de World Health Organization, 202026).

SARS-CoV-2: coronavirus 2 del síndrome respiratorio agudo grave; PCR: reacción en cadena de la polimerasa; EPP: equipo de protección personal.

del sitio www.ancam-imagen.com durante todo el año 2021 y 2022.

Al inicio de la pandemia, muchos departamentos de imagen cardiaca difirieron estudios no urgentes ${ }^{18-20}$ y aplicaron medidas de seguridad e higiene estrictas para disminuir el riesgo de transmisión ${ }^{18-20,23}$.

Ante el riesgo alto y sostenido para enfermar por COVID-19 del personal de salud en México observado en $2020^{2}$ y hasta disponer de medicamentos antivirales más efectivos o alcanzar la cobertura de vacunación necesaria, se debería dar mayor difusión a la prevención primaria contra el riesgo de infección en los profesionales que realizan estudios de imagen cardiaca, desde que se solicita cada prueba hasta que el paciente sale del gabinete. Es previsible la disminución en la intensidad de la pandemia en la primavera, pero 
Tabla 2. Cuestionario de riesgo COVID-19. Para candidato a estudio, familiar o acompañante. Enviar y obtener respuestas por WhatsApp, Telegram, Messenger o correo electrónico, al agendar la cita, antes de que el paciente llegue al servicio de imagen

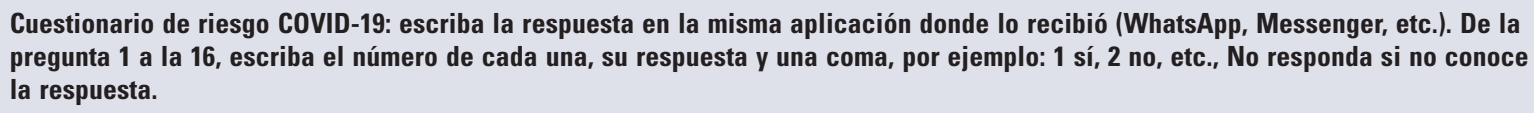

Nombre:

Fecha:

Peso:

Estatura:

Correo electrónico:

Edad:

Fecha de nacimiento:

Hombre Mujer

¿Usted es el paciente o acompaña al paciente al estudio?

Paciente:

Acompañante:

Nombre del médico que envía: Teléfono del médico:

Nombre del estudio solicitado por su médico:

Motivo del estudio:

Fecha de su estudio: Hora: Teléfono:

Paciente externo: use celular y mensajes SMS, WhatsApp, Telegram, etc., Llenar y enviar antes del día de su cita.

1 ¿Tiene infección confirmada por COVID-19 o es sospechoso de tenerla?

2 ¿En los últimos 15 días ha estado en casas, hospitales o sitios con enfermos o portadores conocidos de COVID-19?

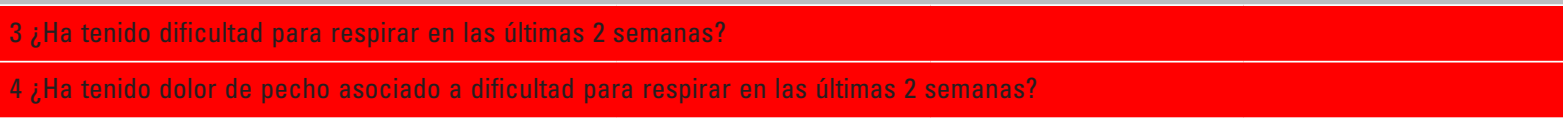

5 ¿Ha tenido tos intensa o frecuente en las últimas 2 semanas?

6 ¿Ha tenido fiebre en las últimas 2 semanas?

7 ¿Ha tenido dolor de cabeza en las últimas 2 semanas?

8 ¿Ha tenido o tuvo gripe en las últimas 2 semanas?

9 ¿Ha tenido dolor $\mathrm{o}$ ardor de garganta en las últimas 2 semanas?

10 ¿Ha tenido dolor de cuerpo o de huesos en las últimas 2 semanas?

11 ¿Ha tenido inflamación de articulaciones o dificultad para su movimiento en los 15 últimos días?

12 ¿Ha tenido ardor o enrojecimiento de ojos en las últimas 2 semanas?

13 ¿Ha tenido congestión nasal en las últimas 2 semanas?

14 ¿Ha tenido diarrea, náuseas o vómitos en las últimas 2 semanas?

15 ¿Ha dejado de percibir olores en las últimas 2 semanas?

16 ¿Ha dejado de percibir el sabor de los alimentos en las últimas 2 semanas?

17 Marque cuál (es) de los siguientes factores de riesgo tiene, si fuera el caso:

$\square$ Tabaquismo $\square$ Hipertensión $\square$ Diabetes $\square$ Obesidad $\square$ Infarto $\square$ Insuficiencia cardiaca $\square$ Cáncer

$\square$ Enfermedad renal $\square$ Hepática $\square$ Hematológica $\square$ Inmunológica $\square$ Enfisema.

Anote si tiene otra enfermedad:

$18 \mathrm{Si}$ el paciente tiene y sabe usar un oxímetro: ¿cuál fue su saturación arterial de oxígeno MíNIMA en las últimas 2 semanas? Anótela aquí: $\%$.

La siguiente información la recopilará el personal técnico del servicio de imagen:

Temperatura inmediata antes de iniciar el estudio de imagen: ${ }^{\circ} \mathrm{C}$

Saturación arterial de oxígeno inmediata antes de iniciar el estudio: $\%$.

Color gris: acudir a evaluación al hospital COVID más cercano a su domicilio si aún no se atiende. Diferir o reagendar estudio.

Color rojo: el médico puede o no hacer algunas preguntas para discriminar entre etiología cardiovascular o respiratoria; ante sospecha de esta última, enviar a urgencias del hospital COVID más cercano y diferir el estudio.

Color naranja: probabilidad alta de portar COVID-19; diferir estudio.

Color amarillo: probabilidad moderada de portar COVID-19. Avise a su médico de cualquier respuesta afirmativa. 
Tabla 3. Logística para hacer las citas por parte del servicio de recepción antes de que el paciente llegue al gabinete Sobre la información que se puede enviar al paciente al agendar una cita a estudio de imagen

1. Cuando se solicite un estudio al servicio de imagen, envíe el cuestionario de riesgo al paciente 0 a la persona responsable del mismo, por Messenger, Telegram, WhatsApp o correo electrónico, desde una computadora o teléfono móvil del servicio de recepción de citas del gabinete; si es posible, evite el papel impreso, un fómite potencial. El cuestionario de riesgo debe llenarse por el paciente y ser devuelto al gabinete a la brevedad, por la misma vía electrónica, para identificar sospecha o enfermedad por COVID-19 antes de que el paciente arribe al gabinete 0 , si está hospitalizado, antes de que acuda al servicio de imagen. Notificar las respuestas al médico responsable de realizar el estudio, quien podrá diferirlo o no, al valorar su indicación y urgencia contra el riesgo de infección del personal.

2. Una hoja con información general para el paciente y su acompañante como la mostrada en la tabla 5; puede enviarse vía electrónica junto con el cuestionario de riesgo, al hacer la cita para estudio, antes de que el paciente acuda al servicio. Es un documento modificable de acuerdo con las necesidades de cada centro.

3. La preparación específica de cada estudio se puede enviar por la misma vía digital, al agendar la cita.

4. La hoja de consentimiento informado por razones de validez jurídica no se debe enviar vía electrónica, se le entrega al paciente al llegar al departamento de imagen para su firma. Use espray con alcohol en el bolígrafo y manos del paciente antes y después de la firma del documento. Trate a la hoja de consentimiento como potencial fómite.

5. Los 3 primeros documentos pueden enviarse vía electrónica como archivo PDF, Word, mensaje de texto o plantilla. Hacer el envío simple, rápido y práctico, de las redes sociales del gabinete al paciente y viceversa.

6. En los casos en que el paciente o su familia no tengan acceso a una red social por teléfono celular o computadora para el envío de los formularios, se realizará el trámite de manera rápida al momento de agendar la cita. El personal que auxilia en el interrogatorio debe usar siempre el equipo de protección correspondiente.

Consideraciones ante el riesgo de infección con enfermos COVID-19 confirmados, sospechosos o asintomáticos:

1.- Enfermos confirmados o bajo sospecha de COVID-19

El riesgo de infección para el personal de salud es mayor al beneficio y diferir el estudio en la mayoría de los pacientes es correcto, hasta que no represente mayor riesgo de contagio. Paciente no intubado: debe usar mascarilla N-95 o P100 y careta.
2.- Paciente asintomático, incluso con resultado PCR-COVID-19 negativo, durante el periodo activo de la pandemia, en el servicio de imagen debe portar:

Cubrebocas SIN VÁLVULA, quirúrgico, de tela tricapa o N95/ P100/ FFP2/ P2/ DS/ KN95, careta. Seguir recomendaciones de higiene para disminuir riesgo de contagio.

\section{La urgencia en imagen cardiaca es poco frecuente.}

\section{Recuerde:}

A.- Trate a todo paciente como posible causa de contagio, aunque no tenga síntomas. La posibilidad de que el paciente sea portadortransmisor asintomático o presintomático de COVID-19 es una realidad. ANTEPONER LA SEGURIDAD DEL PERSONAL DE SALUD SIEMPRE.

B.- Todo paciente con COVID-19 asintomático, diagnosticado o no, puede estornudar, toser, gritar o reír y expulsar aerosoles.

C.- La tasa de resultados falsos negativos de la prueba PCR para COVID-19 aún es alta.

D.- El cubrebocas sin válvula y la careta en el paciente no intubado limitan el peligro de contagio por la expulsión de secreciones y aerosoles de vías aéreas en medio ambientes cerrados como las salas de imagen.

E.- Todo el personal en el servicio de imagen debe usar el cubrebocas recomendado, sin válvula, gafas y el EPP cuando este está indicado.

Figura 2. Consideraciones ante el riesgo de infección con enfermos COVID-19 (adaptada de Choi, et al.,202018; Skali, et al., 202019; Society for Cardiovascular Magnetic Resonance, 202020; Centers for Disease Control and Prevention, 202028,30,31; World Health Organization, 202029; U.S. Food and Drug Administration, 202032; Sickbert-Bennett, et al., 202033). COVID-19: enfermedad por coronavirus 2019; PCR: reacción en cadena de la polimerasa; EPP: equipo de protección personal.

el riesgo de nuevos picos puede seguir al menos un año más. Algunas de las tablas y figuras siguientes se han realizado para difundirse de forma digital y así aprovechar la existencia en 35 millones de hogares ${ }^{24}$ de 80.9 millones de teléfonos móviles con potencial acceso a las redes sociales de Internet en México ${ }^{25}$. 
Tabla 4. Medidas de seguridad e higiene laboral

Medidas de seguridad e higiene laboral

1. Verifique diario antes de iniciar labores, existencia de suficiente agua, jabón y frascos dispensadores de gel con alcohol etílico 0 espray con alcohol al $70 \%$ para personal y pacientes.

2. Verificar que el personal de limpieza disponga de todo lo necesario para su trabajo, que incluye entre otros: atomizadores con alcohol de $70^{\circ} \mathrm{G}$.L., recipientes con $20 \mathrm{ml}$. de hipoclorito de sodio (cloro) por litro de agua y jabón.

3. Al inicio de turno, medir temperatura a todo el personal. Si existe sospecha de enfermedad avise al comité de infecciones, mida saturación de oxígeno y valore uso de TAC simple de tórax por su excelente valor predictivo negativo.

4. Mida temperatura y ofrezca gel alcohol $70^{\circ}$ a toda persona que entre al departamento de imagen, vigile que entren con mascarillas sin válvula y evite acceso a quien no traiga cubrebocas.

5. Empleados sospechosos de COVID-19 no graves: no deben acudir a laborar, si se descarta infección por SARS-CoV-2 con PCR y clínica, pueden reiniciar labores después de 72 horas sin fiebre y sin tratamiento antipirético; si se confirma la enfermedad: deben aislarse en casa y recibir atención médica. Hacer prueba PCR a contactos del trabajador enfermo; avisar al comité de infecciones del centro de trabajo. Para reincorporarse a laborar deben pasar 10 días aislados en casa desde el inicio de los síntomas, más 72 horas sin fiebre y sin uso de antipiréticos. No es necesario realizar una segunda prueba PCR antes de reincorporarse a trabajar después de cumplido este tiempo. Ver cuadro 1. Empleado enfermo asintomático: esperar 10 días desde la realización de la PCR para volver a laborar. Casos graves: evaluar de forma individual el contexto de cada paciente, por lo común deben pasar al menos 20 días desde el inicio de los síntomas, más 72 horas sin fiebre ni uso de antipiréticos para que pueda volver al trabajo figura 1.

6. Planear esquema de coberturas por ausentismo laboral por enfermedad en el centro de imagen. Asegurar el respaldo de médicos expertos para cubrir ausencia laboral en cada área de imagen, implementar tele radiología para trabajar desde casa en la medida de lo posible.

7. Anotar en la hoja de consentimiento informado la lista de todo el personal técnico y médico en contacto con cada paciente confirmado o sospechoso COVID-19, que se atienda en el centro de imagen.

8. Después del diagnóstico de laboratorio confirmatorio de un caso COVID-19 que se haya atendido en el servicio de imagen, el personal médico debe vigilarse los siguientes 14 días después de su última exposición al caso confirmado.

9. Adiestrar al personal administrativo, de limpieza y técnico sobre las mejores prácticas de higiene; incluya la enseñanza sobre el uso correcto de cubrebocas SIN VÁLVULA Y CORRECTAMENTE COLOCADO, uso de gafas y caretas, recomendar al personal portar alcohol gel o atomizador de bolsillo con alcohol de $70^{\circ}$ para aplicar en sus manos y superficies de trabajo, muebles y objetos, antes y después de tocarlos, con especial atención a estetoscopios, baumanómetros, termómetros, oxímetros, teléfonos, manijas, llaves de lavabos y de baños, perillas y barras de las puertas.

10. Toda persona dentro del área de imagen: precaución, evitar contacto con gotas o aerosoles de saliva; cubrebocas y lentes obligatorio; subraye protección ocular por riesgo de contagio; guardar distancia de 2 metros entre personas y recuerde que los aerosoles infectantes viajan más de 2 metros, por tanto, siempre use cubrebocas y lentes. Procure la menor cantidad de personas y permenezca el menor tiempo en interiores. Toser o estornudar cubriendo boca y nariz con el pliegue del codo, no tocar el cubrebocas una vez colocado, si lo hace lavado de manos inmediato. No tocar ojos, nariz ni boca; lavado de manos frecuente. Desechar pañuelos de papel al usarlos y lavar las manos de inmediato.

11. Considere de ser factible, modificar horarios de entrada y salida para evitar aglomeración en el checador. Guardar siempre distancia de dos metros con otras personas.

12. Asigne a varios miembros del equipo médico en su centro hospitalario o gabinete la revisión e incorporación de actualizaciones que publiquen las instancias reguladoras de salud de calidad, como CDC, OMS y sociedades médicas especializadas del área. COVID-19 es una pandemia de rápida evolución, incorporar el nuevo conocimiento generado.

13. No diferir interpretaciones, entregar rápido, el mismo día. En pacientes positivos COVID-19 use medios digitales, en el reporte responda las preguntas clave y entregue imágenes relacionadas con el motivo del estudio.

14. Durante cualquier semáforo que no sea verde en la pandemia COVID-19, al realizar e interpretar el estudio, investigue y responda solo el asunto específico que necesita conocer el médico de referencia, para disminuir el tiempo de estudio y el contacto del personal con el paciente.

15. Favorecer que los médicos de imagen respondan dudas del médico de referencia por redes sociales o teléfono.

16. Evitar que el personal no adiestrado en el manejo de material infecto contagioso ni en el uso correcto de equipo de protección personal (EPP), tenga contacto con pacientes sospechosos o confirmados portadores de COVID-19.

17. Promover en el personal peso ideal, dieta saludable, ejercicio, no fumar, limitar ingesta de alcohol y dormir bien, reducir estrés.

18. Tener presente que cualquier persona puede ser transmisor asintomático o portador presintomático.

Adaptada de European Centre for Disease Prevention and Control, 2020 ; Centers for Disease Control and Prevention, 202010,11; Queensland Health Hospital and Health Service, 202012; NHS England, 202013; American College of Cardiology, 202014; Choi, et al., 202018; Skali, et al., 202019; Society for Cardiovascular Magnetic Resonance, 202020; World Health Organization, 202026; Centers for Disease Control and Prevention, 202027,30,31; U.S. Food and Drug Administration, 202032; Sickbert-Bennett, et al., 202033. 
Tabla 5. Ejemplo de recomendaciones para enviar al paciente

Ejemplo de recomendaciones para enviar al paciente antes de que acuda a su estudio

- Antes de acudir a su estudio, lea con atención este mensaje, las indicaciones de preparación y llene y regrese a la brevedad el cuestionario de riesgo COVID-19 que le ha sido enviado.

- Según el resultado de su cuestionario de riesgo COVID-19, su estudio podría reagendarse varias semanas, de acuerdo con las reglas oficiales de seguridad sanitaria para esta clase de pruebas.

- Es obligatorio el uso de cubrebocas quirúrgico o casero de 3 telas, no traiga cubrebocas con válvula, estos arriesgan al personal médico que lo atenderá. No se retire su cubrebocas dentro del gabinete.

- Use lentes protectores y de forma opcional careta plástica.

- Debe traer gel de alcohol etílico al $70 \%$ o un frasco atomizador con alcohol de $70^{\circ} \mathrm{GL}$ para sus manos.

- Al acudir al centro de imagen se le tomará temperatura y a juicio del médico, saturación arterial de oxígeno.

- Dentro del centro de imagen se han tomado todas las precauciones para que usted esté seguro, como la limpieza frecuente de las instalaciones e instrumentos antes y después de realizar cada estudio, evitar sobreposición de estudios y aglomeración de pacientes a la misma hora; el personal de salud que lo atenderá sigue reglas de seguridad sanitaria profesional para cuidar su salud; el personal técnico o médico se mantendrá a dos metros o más de usted el mayor tiempo posible y cuando se acerque será solo para acciones necesarias, como administrar medicamentos o canalizar una vena y siempre lo hará con cubrebocas y con la protección complementaria adecuada para cada situación clínica. Su estudio se ha adaptado para que usted permanezca el menor tiempo posible y con gran seguridad en nuestro servicio, sus resultados le serán enviados vía electrónica a usted y a su médico tratante. No entregamos su estudio en sobres ni CD ni en papel, para disminuir el riesgo de infección por medio de ese tipo de objetos.

Las medidas descritas son para su seguridad y el éxito también depende de su propia conducta, recuerde por favor:

- Conservar distancia de 2 metros o más con las demás personas en las instalaciones del centro de imagen u hospital. Si se sienta, vigile que siempre exista un asiento vacío a ambos lados, al frente y atrás de usted, para mantener la distancia segura.

- Durante su estancia limite su conversación solo a aspectos necesarios y no eleve la voz, salvo que sea necesario.

- Responda de forma breve, clara y precisa las preguntas que le haga el personal.

- Si tose o estornuda, aunque tenga cubrebocas, cubra su nariz y boca con el pliegue de su codo, evite tocarse nariz, ojos y boca. Después de usar pañuelo o papel desechable, tírelo en los recipientes para basura, no lo guarde y lave enseguida sus manos.

- Si usa su celular, después lave sus manos y aplique alcohol en espray o alcohol-gel.

- Limite el contacto de sus manos con superficies, muebles y aparatos en el gabinete, si lo hace lave sus manos y aplíquese alcohol después. - Acuda con un solo familiar o acompañante o solo usted.

- Para pagar su estudio, prefiera servicios de banca electrónica antes de su cita; si trae dinero en efectivo, traiga solo lo necesario; aplique antes en su casa, alcohol etílico de $70^{\circ}$ en espray a billetes y monedas, déjelos secar, luego guárdelos en una bolsa de plástico cerrada - EI SARS-CoV-2 puede permanecer por horas en el aire de espacios cerrados o mal ventilados; el virus viaja con los aerosoles de saliva expulsados al hablar, reír, toser o estornudar; el contagio ocurre en espacios cerrados con mucha facilidad y a través de mucosas de su boca, nariz y ojos; por tanto, para mantener su riesgo al mínimo, no se quite su cubrebocas ni lentes en el servicio y limite su estancia al menor tiempo posible.

SARS-CoV-2: coronavirus 2 del síndrome respiratorio agudo grave.

Personal de imagen en contacto con paciente sospechoso o portador de COVID-19 asintomático o con síntomas, intubado o no, estable o inestable:

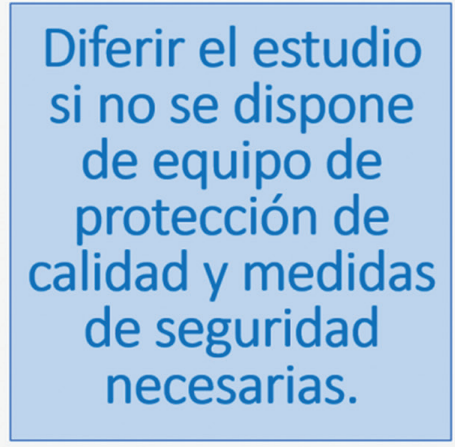

PERSONAL: Uso de EPP apropiado: Cubrebocas sin válvula N95/P100/FFP2/P2/DS/KN95 o equivalente. Protección ocular con lentes especiales, herméticos. Careta protectora, guantes y EPP.

ALGUNAS ACCIONES GENERALES EN LOS SERVICIOS DE IMAGEN CARDIACA ANTE COVID-19

Figura 3. Algunas medidas de prevención para el personal de imagen en contacto con un enfermo COVID-19 (adaptada de Choi, et al.,202018; Skali, et al., 202019; Society for Cardiovascular Magnetic Resonance, 202020).

COVID-19: enfermedad por coronavirus 2019; EPP: equipo de protección personal. 
Tabla 6. COVID-19: acciones generales en el servicio de imagen

COVID-19: acciones generales en el servicio de imagen

1. Reagendar estudios no urgentes en la fase aguda de la pandemia.

2. Trate a todo paciente como potencial portador de COVID-19, tenga o no síntomas.

3. En portadores de comorbilidades para COVID-19 (adultos mayores de 60 años, diabetes, hipertensión arterial, obesidad, tabaquismo, inmunocompromiso, nefropatías o neumopatías crónicas, inmunocomprometidos, patología de oncocardiología, etc.) evaluar riesgo/ beneficio del estudio y posible diferimiento. Valore asignar horario exclusivo para ellos o un día para atenderlos.

4. Cada estudio debe ser realizado de forma segura para el paciente y personal del gabinete.

5. Para atender pacientes con sospecha o portadores de COVID-19: citarlos al final de cada turno de trabajo diario. Considere ampliar horarios entre pacientes, evite citas simultáneas. Cubra los monitores y teclados con películas plásticas desechables.

6. Elaborar lista diaria con el material y equipo que se necesitará antes de iniciar labores y verifique su disponibilidad.

7. Disponga de mayor tiempo para el aseo después de cada estudio: deje sola y cerrada la sala usada para permitir precipitación de algunos aerosoles por 15 a 30 minutos, use 30 a 60 minutos según el tamaño de la instalación, para aseo por personal de limpieza protegido. Use robot de luz UV si dispone de este, como complemento al aseo regular. Valore después permitir ventilación según tipo de paciente atendido. Los aerosoles no se precipitan durante horas en salas cerradas y pueden viajar grandes distancias; cuide tener buena ventilación e intercambio aéreo en sus instalaciones con los filtros y protecciones de acuerdo con las normas sanitarias oficiales.

8. Se deberá tener un área establecida como blanca para vestido cuando se requiere uso de EPP completo, con señalización adecuada y con lo necesario para facilitar el proceso: alcohol gel, cestos marcados para ropa o basura, tarjas con agua y jabón, infografías visibles con secuencia de pasos a seguir. En caso de contaminación mayor, la ruta de acceso a la regadera más cercana debe estar marcada y ser visible con facilidad.

9. Elimine el material desechable siguiendo las normas establecidas de seguridad por el comité de infecciones local, evite contaminar superficies y auto contaminación; limpie y descontamine las sala, material no desechable y aparatos que se hayan utilizado, electrodos, pantallas, teclados, superficies que hayan entrado en contacto con el paciente, teléfono fijos, bombas de infusión, etc., por personal de limpieza entrenado y protegido con EPP.

10. Después de atender a un paciente con sospecha o confirmación COVID-19 productor de aerosoles y que por cualquier razón no porte protección adecuada de vías aéreas, considere limpieza y desinfección de los sistemas de ventilación, siga recomendaciones del comité hospitalario de infecciones y de la autoridad sanitaria oficial.

11. De ser posible, usar telemedicina para interpretar a distancia estudios de imagen. Use conexiones remotas para compartir o interpretar las imágenes. Evite la revisión de las imágenes en persona dentro del gabinete y comparta las imágenes desde las pantallas de la estación de trabajo, comuníquese con los médicos tratantes vía telefónica o redes sociales.

12. Si su centro de imagen suspendió labores durante la etapa aguda de la pandemia, éste se debe reabrir cuando las autoridades de salud federales y locales lo indiquen, siguiendo todas las recomendaciones oficiales sanitarias.

13. Al reiniciar estudios de imagen no urgentes, pasada la fase aguda de la pandemia o después de cuarentena:

a) Seguir las indicaciones de las autoridades sanitarias oficiales y de su centro de trabajo.

b) Disponer de equipo de protección y personal técnico y médico suficiente.

c) En hospitales, debe haber camas disponibles para ingresar al paciente atendido en imagen si se presenta una urgencia.

d) Los servicios de cateterismo, terapia intensiva y hospitalización general, laboratorio de análisis clínicos y de gabinete deben estar disponibles al tiempo de reiniciar estudios no urgentes de imagen.

COVID-19: enfermedad por coronavirus 2019; SPECT: tomografía computarizada por emisión de fotón único; PET: tomografía por emisión de positrones; TC: tomografía computarizada; RM: resonancia magnética.

Adaptada de European Centre for Disease Prevention and Control, 2020 ; Centers for Disease Control and Prevention, 202010,11; Queensland Health Hospital and Health Service, 202012; NHS England, 202013; American College of Cardiology, 202014; Choi, et al.,202018; Skali, et al., 2020 ${ }^{19}$; Society for Cardiovascular Magnetic Resonance, 202020; World Health Organization, 202026; Centers for Disease Control and Prevention, 202027.

Debido a la gravedad y duración del brote pandémico, muchos centros de diagnóstico podrían difundir, adaptar y modificar esta información en razón a sus características, recursos y leyes de salud vigentes. La información aquí descrita se basa en las mejores prácticas de higiene, seguridad laboral y sanitaria publicadas a la fecha ${ }^{26-33}$ y se presentan en su mayor parte mediante tablas (tablas 1 a 6 ) y en figuras (figuras 1 a 9), para su difusión a través de redes sociales entre el personal de salud; algunas podrían ser enviadas a los pacientes, como la tabla 2, que ejemplifica un cuestionario de riesgo de COVID-19 para el paciente antes de que este acuda al estudio de imagen. Se pueden enviar al momento en que se solicita la prueba, basta un teléfono celular y una red social como WhatsApp, Telegram, Messenger, etc., 


\section{Antes del estudio:}

*Responder cuestionario de riesgo COVID-19 (tabla 2), anotar temperatura y $\mathrm{SaO} 2$ por oximetría digital.

* Reagendar estudio si el cuestionario de riesgo muestra sospecha o confirma COVID-19, previa consideración riesgo beneficio por diferir la prueba y notifique a su médico.

*Lavado de manos con agua y jabón. Opcional: gel / espray de alcohol etílico $70^{\circ}$.

* Permanecer con mascarilla quirúrgica y lentes (la careta plástica NO sustituye a los lentes) durante todo el estudio.

*Minimice tiempos de espera en áreas comunes de acceso. *Si descubre que un paciente es sospechoso o portador COVID-19 pasarlo al final del turno. Si por cualquier razón este pasa antes a estudio, realizar después aseo exhaustivo con tiempo de espera de 15 a 30 minutos para permitir recambio aéreo de la sala.

Siempre tener presente que:

\section{Durante el estudio:}

Minimice tiempos de estudio, prefiera protocolos rápidos y evite e esfuerzo físico. Pida al paciente no tocar nada y hablar sin elevar la voz. En TC cardiaca tome primero TC simple de tórax.

Después del estudio:

RESULTADO: Entréguelo de inmediato, envíelo vía electrónica, use redes sociales y correo electrónico. Evite que el paciente lo espere en el hospital.

Considere 15 a 30 minutos sin personal dentro del área donde se realizó el estudio, permita intercambio aéreo.

Los aerosoles duran horas suspendidos en áreas cerradas sin ventilación; después use 30 a 60 minutos para limpieza completa por el personal designado debidamente protegido.

Reagende todo estudio si no hay equipo de protección personal para manejar pacientes productores de aerosoles.

Durante la pandemia de COVID-19 se han hecho y se seguirán haciendo estudios de imagen a pacientes portadores de COVID-19 asintomáticos no diagnosticados, incluso con PCR falsa negativa, este fenómeno probablemente durará la mayor parte del año 2021 y parte del 2022 . Los aerosoles infectantes con SARS CoV-2 suspendidos en el aire de espacios cerrados parecen ser la forma más importante de contagio para el personal de imagen. Priorice uso de cubrebocas sin válvula, lentes protectores y detecte pacientes de riesgo ANTES de su arribo a estudio.

PACIENTE ASINTOMÁTICO DE BAJA PROBABILIDAD O COVID-19 NEGATIVO, SIN SOSPECHA DE ENFERMEDAD.

Figura 4. Logística en el paciente asintomático, COVID-19 negativo o de baja probabilidad, con cuestionario de riesgo negativo (adaptada de Choi, et al.,202018; Skali, et al., 202019; Society for Cardiovascular Magnetic Resonance, 202020). COVID-19: enfermedad por coronavirus 2019; SA02: saturación de oxígeno; SARS-CoV-2: coronavirus 2 del síndrome respiratorio agudo grave; PCR: reacción en cadena de la polimerasa; TC: tomografía computarizada.
Intubado
Difiera el estudio hasta mejorar condiciones críticas y el médico de referencia revalore.
Situaciones de excepción son raras; el riesgo de contagio para el personal suele ser mayor al beneficio de cualquier estudio de imagen en paciente crítico COVID-19.

No intubado

Reagende el estudio si no se pone en peligro la vida por diferir la prueba de imagen. Avise al médico de referencia.

Todo paciente: reagende si el personal no tiene el equipo de protección oficial para tratar pacientes productores de aerosoles.

Si se decide realizar, siempre tener presente:

* Elimine tiempos de espera en áreas comunes y de acceso. Permita solo un acompañante. Haga el estudio al final del turno.

* Programar llegada directa del enfermo a sala de estudio con todas las medidas de seguridad para disminuir riesgo de contagio.

* Durante el trayecto a sala de estudio acercarse a menos de dos metros del enfermo solo con mascarilla N95 y EPP, gafas y careta.

* Si el paciente está hospitalizado, sincronice cada momento desde la recepción del paciente hasta su salida del servicio.

* Es obligada la vigilancia continua a distancia con monitor de saturación arterial de oxígeno y signos vitales del paciente.

* Equipo IDEAL DESEABLE para personal médico y técnico al entrar a sala de estudio: EPP con traje impermeable tipo Tyvek, mascarilla o respirador N95, lentes protectores, escafandra o careta, bata impermeable, guantes dobles y botas quirúrgicas.

* El paciente no intubado debe usar AL MENOS: mascarilla N95/P100/FFP2/P2/DS o KN95, careta plástica y bata.

* El personal debe usar AL MENOS: mascarilla N95 o similar, lentes protectores y careta, bata impermeable o bata con mandil impermeable, guantes dobles y botas quirúrgicas, durante todo el estudio. Debe saber ponerse y retirar EPP, ver algunas de las técnicas correctas recomendadas por CDC y OMS en material suplementario figuras A, B y C.

* Minimizar tiempos de estudio, prefiera protocolos rápidos y pida al paciente no tocar nada y hablar en voz baja solo si es necesario.

PACIENTE CONFIRMADO O PROBABLE COVID-19 LOGÍSTICA ANTE INDICACIÓN DE ESTUDIO DE IMAGEN.
}

Figura 5. Abordaje y algunas acciones clave en el paciente confirmado o probable COVID-19 antes del estudio de imagen (adaptada de Choi, et al.,202018; Skali, et al., 202019; Society for Cardiovascular Magnetic Resonance, $2020^{20}$ ). COVID-19: enfermedad por coronavirus 2019; EPP: equipo de protección personal; CDC: Centers for Disease Control and Prevention; OMS: Organización Mundial de la Salud. 


\section{Después del estudio:}

- Entregue el resultado a la brevedad vía electrónica, use redes sociales o sistema para enviar formato electrónico si existe en su institución.

- Envíe de inmediato al paciente a su cama si está hospitalizado o a su domicilio si es paciente ambulatorio, evite que el paciente espere en el servicio de imagen su resultado.

- Cierre las áreas DESPUÉS de usadas por 15 a 30 minutos, sin personal dentro, para permitir la precipitación de aerosoles al piso, después use 30 a 60 minutos para hacer aseo completo siguiendo protocolos del gabinete. Durante los picos de la pandemia los sistemas de tomografía pueden estar saturados y los horarios y tiempos de aseo se podrán adaptar a conveniencia de cada gabinete.

- El personal de limpieza debe estar protegido con EPP, N95, guantes, botas, lentes, gorro y careta plástica. Si no se dispone de EPP debe usar todo el equipo restante aquí descrito, bata impermeable o quirúrgica con filipina y pantalón quirúrgico incluidos.

\section{PACIENTE CONFIRMADO O CON SOSPECHA DE ENFERMEDAD ACTUAL POR COVID-19.}

Figura 6. Algunas acciones clave en el paciente confirmado o probable COVID-19 después del estudio de imagen (adaptada de Choi, et al.,202018; Skali, et al., 202019; Society for Cardiovascular Magnetic Resonance, 202020). COVID-19: enfermedad por coronavirus 2019; EPP: equipo de protección personal.

Si existen, seguir las guías de seguridad de su centro de trabajo.

- Antes de atender pacientes COVID-19: el personal de imagen debe recibir un curso de adiestramiento suficiente para aprender a colocar y retirar el EPP y el manejo de residuos infectocontagiosos.
- Lavado de manos con agua y jabón, aplicar gel o espray con alcohol de $70^{\circ}$, vestir EPP antes de atender al paciente. Después, lavado y retiro de EPP siguiendo proceso aprendido y baño, en cada área específica.

- Equipo de protección personal (EPP) impermeable si esta disponible.

- En ausencia de EPP impermeable tipo traje Tyvek, valorar diferir estudio. Si se decide hacer la prueba evaluar en lugar de traje Tyvek el uso de uniforme quirúrgico, (pantalón y filipina) con bata quirúrgica, reforzada con puño. Si la bata no es impermeable añada mandil de plástico.

- Lentes con sellado de bordes a piel de la cara y careta o escafandra.

- Guantes de látex o nitrilo ante alergia al primero, dobles. Selle con tela adhesiva los bordes del guante a la bata o del traje del EPP.

- Mascarilla N 95 o equivalente. Revise que los bordes queden sellados a la piel de la cara, no use barba. No use mascarilla con válvula. Si los lentes se humedecen con su aliento los bordes de la mascarilla tienen fuga, selle entonces con cinta de microporo.

- Gorro desechable.

- Botas desechables.

PUNTOS IMPORTANTES PARA PROTECCIÓN DEL PERSONAL DE IMAGEN QUE ATIENDE PACIENTES PRODUCTORES DE AEROSOLES DE VÍAS RESPIRATORIAS, SOSPECHOSOS O CONFIRMADOS PORTADORES DE COVID-19.

Figura 7. Medidas principales de seguridad en personal que trata pacientes que producen aerosoles, con sospecha o portadores confirmados de COVID-19 (adaptada de Choi, et al.,202018; Skali, et al., 2020 ${ }^{19}$; Society for Cardiovascular Magnetic Resonance, $2020^{20}$ ).

COVID-19: enfermedad por coronavirus 2019; EPP: equipo de protección personal. 
Antes de que el paciente ingrese a sala de imagen:

- Cubra computadoras, teclados, máquinas y muebles dentro del área de estudio con plástico transparente desechable, fije con cinta adhesiva.

- Cubra con plástico, tela quirúrgica desechable o sábanas el equipo que no se usará.

- Preferir sistemas de aire acondicionado con filtros ULPA o HEPA con sellado de gel.

- Siga todas las recomendaciones de las autoridades sanitarias de su hospital o gabinete de imagen, establecidas para el manejo y desecho de ropa y equipo de protección usado en cada paciente.

- El personal de limpieza debe estar preparado y protegido con EPP o traje y bata quirúrgicos impermeables o con mandil plástico; usar mascarilla N95 o equivalentes, guantes, botas, gafas protectoras y careta.
Después de que sale de la adquisición de su estudio de imagen:

- Retire las barreras de plástico y sábanas de la habitación, colóquelas en una bolsa impermeable desechable, selle y etiquete: "RIESGO: COVID-19", Ilévela a lavandería o al recipiente de desechos biológicos, según el caso.

- De ser posible cierre la sala después de cada estudio por 15 a 30 minutos. Los aerosoles suspendidos en el aire de espacios cerrados representan un potencial riesgo de contagio, cite a los pacientes con sospecha o conocidos COVID-19 al final del turno.

- El personal de limpieza debe lavar toda el área y equipos usados, pisos, paredes, escáner, instrumental y muebles incluidos; usar jabón o detergente común o desinfectante activo contra agentes patógenos víricos, como el hipoclorito al 20/1000 (20 ml de cloro en $980 \mathrm{ml}$ de agua). Los cables de electrodos, transductores, teclados y pantallas pueden dañarse con el cloro; usar alcohol de $70^{\circ}$.

- Tras la limpieza de la sala realizar aseo de los espacios donde estuvo el paciente infectado, el uso de robot de luz ultravioleta debe ser medida adicional, no el único medio de desinfección.

PUNTOS CLAVE SOBRE LIMPIEZA ANTES Y DESPUÉS DE ATENDER A PACIENTE SOSPECHOSO O CON COVID-19.

Figura 8. Limpieza antes y después de atender en sala de imagen a un paciente con sospecha o portador conocido de COVID-19 (adaptada de Choi, et al.,202018; Skali, et al., 202019; Society for Cardiovascular Magnetic Resonance, 202020). COVID-19: enfermedad por coronavirus 2019; EPP: equipo de protección personal.

Puntos clave de seguridad sanitaria con pacientes COVID-19 en salas de CT, RM, SPECT y PET

\begin{tabular}{|c|}
\hline ANTES DEL ESTUDIO \\
\hline $\begin{array}{l}\text { * Verificar cuestionario de } \\
\text { riesgo, considere reagendar } \\
\text { ante sospecha o } \\
\text { confirmación de COVID-19. }\end{array}$ \\
\hline * Verificar limpieza de sala. \\
\hline $\begin{array}{c}\text { * Asegurar disponer todo el } \\
\text { material e insumos } \\
\text { necesarios. }\end{array}$ \\
\hline $\begin{array}{l}\text { * Película plástica: teclados, } \\
\text { pantallas y controles. }\end{array}$ \\
\hline $\begin{array}{c}\text { * Asegurar ruta al gabinete } \\
\text { de imagen. }\end{array}$ \\
\hline
\end{tabular}

*

\section{DURANTE EL ESTUDIO}

* Solo personal indispensable.

*Ruta de acceso y salida definida.

*Usar EPP y limitar tiempo de estancia.

*Diferir pruebas de esfuerzo físico, aún casos urgentes.

* Evitar dispersar aerosoles, puertas cerradas durante el estudio.

* Sustituir pruebas de esfuerzo físico por estimulación farmacológica, hasta final de pandemia.
DESPUÉS DEL ESTUDIO

*Salas de CT, RM, PET, SPECT: 15 a 30 minutos sin personal, puertas cerradas.

*Aseo exhaustivo por personal de limpieza protegido con EPP.

*Usar agua y jabón, alcohol de $70^{\circ}$, hipoclorito de sodio 20/1000: $20 \mathrm{ml}$ de cloro y $980 \mathrm{ml}$ de agua.

*El robot de rayos UV puede usarse adicional a la limpieza descrita.

EPP: Equipo de protección personal, incluir siempre mascarilla N-95 o similar*, gafas herméticas, gorro, careta, filipina, bata y pantalón quirúrgicos, traje impermeable Tyvek, guantes de látex o nitrilo y botas desechables. Si no hay traje impermeable, usar bata impermeable o bata quirúrgica con mandil plástico y el resto del equipo descrito.

*PREFIERA MASCARILLAS Y RESPIRADORES CUYA EFICIENCIA HA SIDO EVALUADA POR LOS C.D.C.

Figura 9. Puntos clave de seguridad sanitaria en salas de imagen con pacientes COVID-19 (adaptada de Choi, et al.,202018; Skali, et al., 202019; Society for Cardiovascular Magnetic Resonance, 202020).

COVID-19: enfermedad por coronavirus 2019; SPECT: tomografía computarizada por emisión de fotón único; PET: tomografía por emisión de positrones; TC: tomografía computarizada; RM: resonancia magnética; CDC: Centers for Disease Control and Prevention. 
que son de uso amplio, simple y práctico y se prefieren al correo electrónico, pues son más rápidas. Cuando el paciente no disponga de redes sociales ni correo electrónico se puede aplicar vía telefónica o al arribo del paciente al gabinete. El contenido de las tablas se puede adaptar al nivel cultural de cada paciente y a las condiciones de cada centro de imagen.

\section{Conclusiones}

Algunas de las presentes recomendaciones no se han probado de forma sistemática y se aplican bajo una base teórico-práctica. Pueden variar ante nuevos hallazgos, se basan en la información inicial del primer año con la enfermedad y no sustituyen el juicio clínico del médico. El acceso a la vacuna puede ser complejo y tardado en el contexto nacional actual. Un reciente avance contra la pandemia en México es que buena parte del personal médico de primera línea que atiende enfermos sintomáticos de COVID-19 ya recibió una o dos dosis de alguna de las vacunas de reciente acceso, pero la mayoría de los profesionales sanitarios de imagen cardíaca en toda la nación no han sido vacunados al mes de marzo de 2021 y no están clasificados como de primera línea34, aunque a diario están en contacto cercano por muchas horas con pacientes que ignoran ser portadores asintomáticos de SARS-CoV-2 y que representan un riesgo potencial de infección para dicho personal. Es importante mantener las medidas de protección y seguridad descritas en este documento hasta que la pandemia por COVID-19 sea oficialmente declarada terminada por la OMS, puesto que la mutación y surgimiento de nuevas cepas podría llevar a futuros brotes epidémicos; en tanto, la aplicación de este tipo de recomendaciones aumenta la probabilidad de reducir el número de nuevos contagios. La página www. ancam-imagen.com contiene información complementaria útil.

\section{Financiamiento}

La presente investigación no ha recibido ninguna beca específica de agencias de los sectores públicos, comercial o sin ánimo de lucro.

\section{Conflicto de intereses}

Los autores declaran no tener conflicto de intereses.

\section{Responsabilidades éticas}

Protección de personas y animales. Los autores declaran que para esta investigación no se han realizado experimentos en seres humanos ni en animales.

Confidencialidad de los datos. Los autores declaran que han seguido los protocolos de su centro de trabajo sobre la publicación de datos de pacientes.

Derecho a la privacidad y consentimiento informado. Los autores declaran que en este artículo no aparecen datos de pacientes.

\section{Bibliografía}

1. Organización Mundial de la Salud. Cronología de la respuesta de la OMS a la COVID-19 [Internet]. Organización Mundial de la Salud [consultado: 1 de enero de 2021]. Disponible en: https://www.who.int/es/news-room/ detail/29-06-2020-covidtimeline

2. Amnistía Internacional. Global: Análisis de Amnistía Internacional revela que más de 7 mil personas trabajadoras de la salud han muerto a causa de COVID-19 [Internet]. Amnistía Internacional [consultado: 3 de septiembre de 2020]. Disponible en: https://amnistia.org.mx/contenido/ index.php/global-analisis-de-amnistia-internacional-revela-que-mas-de-7-mil-personas-trabajadoras-de-la-salud-han-muerto-acausa-de-covid-19/

3. Bernheim A, Mei X, Huang M, Yang Y, Fayad ZA, Zhang N, et al. Chest CT findings in coronavirus disease-19 (COVID-19): relationship to duration of infection. Radiology. 2020;295:685-91.

4. Clerkin KJ, Fried JA, Raikhelkar J, Sayer G, Griffin JM, Masoumi A, et al. COVID-19 and cardiovascular disease. Circulation. 2020;141: 1648-55.

5. Centers for Disease Control and Prevention. What healthcare personnel should know about caring for patients with confirmed or possible coronavirus disease 2019 (COVID-19) [Internet]. Centers for Disease Control and Prevention [consultado: 3 de diciembre de 2020]. Disponible en: https://www.cdc.gov/coronavirus/2019-ncov/hcp/caring-for-patients-H.pdf

6. Driggin E, Madhavan MV, Bikdeli B, Chuich T, Laracy J, Biondi-Zoccai G, et al. Cardiovascular considerations for patients, health care workers, and health systems during the COVID-19 pandemic. J Am Coll Cardiol. 2020;75:2352-71.

7. Ong S, Tan $\mathrm{Y}$, Chia $\mathrm{P}$, Lee $\mathrm{T}, \mathrm{Ng} \mathrm{O}$, Wong $\mathrm{M}$, et al. Air, surface environmental, and personal protective equipment contamination by severe acute respiratory syndrome coronavirus 2 (SARS-CoV-2) from a symptomatic patient. JAMA. 2020;323(16):1610 -12.

8. van Doremalen N, Bushmaker T, Morris HD, Holbrook MG, Gamble A Williamson BN, et al. Aerosol and surface stability of SARS-CoV-2 as compared with SARS-CoV-1. N Engl J Med. 2020;382:1564-1567.

9. European Centre for Disease Prevention and Control. COVID-19 pandemic [Internet]. European Centre for Disease Prevention and Control [consultado: 11 de agosto de 2020]. Disponible en: https://www.ecdc.europa. eu/en/covid-19-pandemic

10. Centers for Disease Control and Prevention. Steps healthcare facilities can take now to prepare for COVID-19 [Internet]. Centers for Disease Control and Prevention [consultado: 10 de agosto de 2020]. Disponible en: https://www.cdc. gov/coronavirus/2019-ncov/healthcare-facilities/ steps-to-prepare.html

11. Centers for Disease Control and Prevention. Interim guidance for healthcare facilities: preparing for community transmission of COVID-19 in the United States [Internet]. Centers for Disease Control and Prevention [consultado: 20 de Julio de 2020]. Disponible en: https://stacks.cdc.gov/ view/cdc/85502

12. Queensland Health Hospital and Health Service. Interim infection prevention and control guidelines for the management of COVID-19 in healthcare settings [Internet]. Queensland Health Hospital and Health Service [consultado: 25 de octubre de 2020]. Disponible en: https://www.health. qld.gov.au/_data/assets/pdf_file/0038/939656/qh-covid-19-Infection-control-guidelines.pdf

13. NHS England. Coronavirus guidance for clinicians and NHS managers [Internet]. NHS England [consultado: 4 de noviembre de 2020]. Disponible en: https://www.england.nhs.uk/coronavirus/

14. American College of Cardiology. ACC's COVID-19 Hub [Internet]. American College of Cardiology [consultado: 21 de diciembre de 2020. Disponible en: https://www.acc.org/latest-in-cardiology/features/accs-coronavirus-disease-2019-covid-19-hub\#sort=\%40fcommonsortdate90022\%20descending 
15. Elkind MSV, Harrington RA, Benjamin IJ. The role of the American Heart Association in the Global COVID-19 Pandemic. Circulation. 2020; 141:e743-e745.

16. Alcocer-Gamba MA, Gutiérrez-Fajardo $P$, Cabrera-Rayo A, Sosa-Caballero A, Piña-Reyna $Y$, Merino-Rajme JA, et al. Excerpts from the documents of Mexican positions and recommendations in cardiovascular diseases and COVID-19. Arch Cardiol Mex. 2020;90(Supl):10010.

17. Puntmann VO, Carerj ML, Wieters I, Fahim M, Arendt C, Hoffmann J et al. Outcomes of cardiovascular magnetic resonance imaging in patients recently recovered from coronavirus disease 2019 (COVID-19) JAMA Cardiol. 2020;5(11):1265-73.

18. Choi AD, Abbara S, Branch KR, Feuchtner GM, Ghoshhajra B Pontone G, et al. Society of Cardiovascular Computed Tomography guidance for use of cardiac computed tomography amidst the COVID-19 pandemic Endorsed by the American College of Cardiology. J Cardiovasc Comput Tomogr. 2020;14(2):101-4.

19. Skali H, Murthy VL, Al-Mallah MH, Bateman TM, Beanlands R, Better N, et al. Guidance and Best Practices for Nuclear Cardiology Laboratories during the Coronavirus Disease 2019 (COVID-19) Pandemic: An Information Statement from ASNC and SNMMI. J Nucl Cardiol. 2020;27(3):1022-9.

20. Society for Cardiovascular Magnetic Resonance. SCMR's COVID-19 preparedness toolkit [Internet]. Society for Cardiovascular Magnetic Resonance [consultado: 20 de junio de 2020. Disponible en: https://scmr. org/page/COVID19

21. British Society of Thoracic Imaging: COVID-19 Resources [Internet] British Society of Thoracic Imaging [consultado: 3 de noviembre de 2020. Disponible en: https://www.bsti.org.uk/covid-19-resources/

22. Mossa-Basha M, Meltzer CC, Kim DC, Tuite MJ, Kolli KP, Tan BS. Radiology Department preparedness for COVID-19: radiology scientific expert panel. Radiology. 2020;296(2):E106-E112.

23. U.S. Department of Labor Occupational Safety and Health Administration Guidance on Preparing Workplaces for COVID-19 [Internet]. U.S. Department of Labor Occupational Safety and Health Administration [consultado: 1 de enero de 2021. Disponible en: https://www.osha.gov/Publications/OSHA3990.pdf

24. Gobierno de México. Consejo Nacional de Población. La composición de las familias y hogares mexicanos se ha transformado en décadas recientes como resultado de cambios demográficos y sociales [Internet]. Gobierno de México, Consejo Nacional de Población [consultado: 1 de enero de 2021. Disponible en: https://www.gob.mx/conapo/articulos/ la-composicion-de-las-familias-y-hogares-mexicanos-se-ha-transformado-en-las-recientes-decadas-como-resultado-de-cambios-demograficos?idiom $=\mathrm{es}$
25. Burgueño Salas $E$. México: número de usuarios de teléfonos celulares inteligentes 2015-2025 [Internet]. México: STATISTA. [consultado: 20 de noviembre de 2020. Disponible en: https://es.statista.com/estadisticas/1077622/ usuarios-de-smartphone-en-mexico/\#; 'text=M\%C3\%A9xico\%3A\%20n\%C3\%BAmero\%20de\%20usuarios\%20de\%20tel\%C3\%A9fonos\%20celulares\%2Ointeligentes\%202015\%2D2025\&text=En\%202020\%2C\%20el\%20 n\%C3\%BAmero\%20de,los\%2095\%20millones\%20en\%202025

26. World Health Organization. Criteria for releasing COVID-19 patients from isolation [Internet]. World Health Organization [consultado: 1 de enero de 2021. Disponible en: https://www.who.int/news-room/commentaries/detail/criteria-for-releasing-covid-19-patients-from-isolation

27. Centers for Disease Control and Prevention. Discontinuation of transmission-based precautions and disposition of patients with COVID-19 in healthcare settings (Interim Guidance) [Internet]. Centers for Disease Control and Prevention [consultado: 30 de Agosto de 2020. Disponible en: https://www.cdc.gov/coronavirus/2019-ncov/hcp/disposition-hospitalized-patients.html?fbclid=IwAR1RQ37Ugx0lzB7ZlcWu_hzUUe4Womlg6dkk2D1k2GeDUwCDaqcs0bW8ufs

28. Centers for Disease Control and Prevention. Sequence for putting on personal protective equipment (PPE) [Internet]. Centers for Disease Control and Prevention [consultado: 4 de enero de 2021. Disponible en: https://www.cdc.gov/hai/pdfs/ppe/PPE-Sequence.pdf

29. World Health Organization. COVID-19: como ponerse y quitarse el equipo de protección personal (EPP) [Internet]. World Health Organization [consultado: 4 de enero de 2021. Disponible en: https://openwho.org/courses/IPC-PPE-ES

30. Centers for Disease Control and Prevention. NIOSH-Approved Particulate Filtering Facepiece Respirators [Internet]. Centers for Disease Control and Prevention [consultado: 4 de enero de 2021. Disponible en: https://www.cdc.gov/niosh/nppt//topics/respirators/disp_part/default.html

31. Centers for Disease Control and Prevention. NPPTL respirator assessments to support the COVID-19 response [Internet]. Centers for Disease Control and Prevention [consultado: 4 de enero de 2021. Disponible en: https://www.cdc.gov/niosh/nppt//respirators/testing/NonNIOSHresults.htm

32. U.S. Food and Drug Administration. Emergency use authorization [Internet]. U.S. Food and Drug Administration [consultado: 4 de enero de 2021 [Internet]. Disponible en: https://www.fda.gov/emergency-preparedness-and-response/mcm-legal-regulatory-and-policy-framework/emergency-use-authorization\#2019-ncov

33. Sickbert-Bennett EE, Samet JM, Clapp PW, Chen H, Berntsen J, Zeman KL, et al. Filtration efficiency of hospital face mask alternatives available for use during the COVID-19 pandemic. JAMA Intern Med. 2020 Aug 11:e204221. doi: 10.1001/jamainternmed.2020.4221. Online ahead of print.

34. Gobierno de México. Conferencia de Prensa \#COVID19 | 13 de marzo de 2021. consultado el 14 de Marzo de 2014, disponible en: https://www. youtube.com/watch?v=dhJ5wqOeTgU 